Research paper

\title{
Revisiting the Pneumocystis host specificity paradigm and transmission ecology in wild Southeast Asian rodents
}

\author{
Alice Latinne ${ }^{\mathrm{a}, \mathrm{b}, \mathrm{c}, *}$, Hsuan-Wien Chen ${ }^{\mathrm{d}}$, Chi-Chien Kuo ${ }^{\mathrm{e}}$, Renee Lorica ${ }^{\mathrm{f}}$, Grant Singleton ${ }^{\mathrm{f}, \mathrm{g}}$, \\ Alex Stuart $^{\mathrm{f}}$, Fedelino F. Malbas ${ }^{\mathrm{h}}$, Christine Demanche ${ }^{\mathrm{i}}$, Magali Chabé ${ }^{\mathrm{i}}$, Johan Michaux ${ }^{\mathrm{c}, \mathrm{j}}$, \\ Serge Morand ${ }^{\mathrm{k}, 1}$ \\ ${ }^{\text {a } W i l d l i f e ~ C o n s e r v a t i o n ~ S o c i e t y, ~ V i e t ~ N a m ~ C o u n t r y ~ P r o g r a m, ~ H a ~ N o i, ~ V i e t ~ N a m ~}$ \\ ${ }^{\mathrm{b}}$ Wildlife Conservation Society, Health Program, Bronx, NY, USA \\ ${ }^{c}$ Université de Liège, Laboratoire de Génétique de la Conservation, GeCoLAB, 4000 Liège, Belgium \\ ${ }^{\mathrm{d}}$ Department of Biological Resources, National Chiayi University, Chiayi, Taiwan \\ ${ }^{\mathrm{e}}$ Department of Life Science, National Taiwan Normal University, Taipei, Taiwan \\ ${ }^{\mathrm{f}}$ International Rice Research Institute, DAPO Box 7777, Metro Manila, Philippines \\ ${ }^{\mathrm{g}}$ Natural Resource Institute, University of Greenwich, Chatham Maritime, Kent, UK \\ ${ }^{\mathrm{h}}$ Research Institute for Tropical Medicine, Department of Health, Muntinlupa, Metro Manila, Philippines \\ ${ }^{\mathrm{i}}$ Univ. Lille, CNRS, Inserm, CHU Lille, Institut Pasteur de Lille, U1019-UMR 9017-CIIL-Centre d'Infection et d'Immunité de Lille, Lille, France \\ ${ }^{\mathrm{j}}$ Animal Santé Territoire Risque Environnement- Unité Mixe de Recherche 117 (ASTRE) Univ. Montpellier, Centre International de Recherche Agronomique pour le \\ Développement (CIRAD), Institut National de la Recherche Agronomique, 34398 Montpellier, France \\ ${ }^{\mathrm{k}}$ University of Montpellier, Institut des Sciences de l'Evolution, CNRS-IRD, Montpellier, France \\ ${ }^{1}$ University of Kasetsart, Faculty of Veterinary Technology, ASTRE-CIRAD, Bangkok, Thailand
}

\section{A R T I C L E I N F O}

\section{Keywords:}

Pneumocystis

Rodents

Southeast Asia

Muridae

Host specificity

Habitat fragmentation

\begin{abstract}
A B S T R A C T
Pneumocystis fungi are opportunistic parasites of mammalian lungs whose evolution, ecology and host specificity in natural host populations remain poorly understood and controversial. Using an extensive collection of 731 lung samples from 27 rodent species sampled in five Southeast Asian countries, and nested PCR amplification of mitochondrial and nuclear genes, we investigated the host specificity and genetic structure of Pneumocystis lineages infecting wild rodents. We also identified the rodent species playing a central role in the transmission of these parasites using network analysis and centrality measurement and we characterized the environmental conditions allowing Pneumocystis infection in Southeast Asia using generalized linear mixed models.

Building upon an unprecedented Pneumocystis sampling from numerous rodent species belonging to closely related genera, our findings provide compelling evidence that the host specificity of Pneumocystis lineages infecting rodents is not restricted to a single host species or genus as often presented in the literature but it encompasses much higher taxonomic levels and more distantly related rodent host species. The phylogenetic species status at both mitochondrial and nuclear genetic markers of at least three new Pneumocystis lineages, highly divergent from Pneumocystis species currently described, is also suggested by our data. Our models show that the probability of Pneumocystis infection in rodent hosts is positively correlated to environmental variables reflecting habitat fragmentation and landscape patchiness. Synanthropic and habitat-generalist rodents belonging to the Rattus, Sundamys and Bandicota genera played a role of bridge host species for Pneumocystis spreading in these heterogeneous habitats, where they can reach high population densities. These are critical findings improving our understanding of the ecology of these enigmatic parasites and the role played by cospeciation and host switches in their evolution. Our results also confirmed the role of land-use change and habitat fragmentation in parasite amplification and spillover in rodents.
\end{abstract}

\footnotetext{
* Corresponding author at: Wildlife Conservation Society, Viet Nam Country Program, Ha Noi, Viet Nam.

E-mail address: alatinne@wcs.org (A. Latinne).
} 


\section{Introduction}

Pneumocystis organisms are airborne-transmitted fungal parasites that infect the lungs of numerous mammal species and require a living host at each stage of their life cycle (Aliouat-Denis et al., 2008; Cissé and Hauser, 2018). Pneumocystis infection may cause life-threatening pneumonia in immunocompromised individuals but asymptomatic and benign infections are also commonly observed in immunocompetent and healthy hosts (Chabé et al., 2004). Several molecular studies investigating the diversity and host specificity of these fungal parasites in a wide range of mammalian species showed that the Pneumocystis genus is highly diversified and includes numerous divergent taxonomic entities characterized by strong host specificity and apparently restricted to a single host species (Akbar et al., 2012; Danesi et al., 2016; Demanche et al., 2001; Hugot et al., 2003; Petružela et al., 2019). However recent studies called this long-standing Pneumocystis strict host specificity paradigm into question as the biology, evolution, epidemiology and transmission of these airborne-transmitted organisms in natural host populations remain poorly understood (Babb-Biernacki et al., 2020; Cissé and Hauser, 2018; Latinne et al., 2018; Ma et al., 2018).

Pneumocystis infection is common and widespread in both laboratory and wild Old World rodents (Cushion et al., 2004; Danesi et al., 2016; Demanche et al., 2015; Latinne et al., 2018; Palmer et al., 2000; Petružela et al., 2019). To date, only three Pneumocystis species infecting rodents have been formally described. Pneumocystis carinii (Frenkel, 1999 ) is the type species of the genus and was first identified in the lungs of laboratory rats (Rattus norvegicus). Pneumocystis wakefieldiae (Cushion et al., 2004) is the second species described in laboratory rats, while P. murina (Keely et al., 2004) is the sole species described in laboratory mice (Mus musculus). However, recent studies of Pneumocystis parasites infecting rodents from Southeast Asia and Africa revealed a much larger Pneumocystis diversity in wild rodents, including many unknown lineages corresponding to potential new species (Latinne et al., 2018; Petružela et al., 2019). Several of these lineages were clearly delineated in phylogenies based on mitochondrial markers, and the level of mitochondrial genetic distance between these lineages and formally recognized species was indicative of phylogenetic species recognition (Latinne et al., 2018). The use of nuclear markers, notoriously difficult to amplify in samples from wild hosts characterized by low Pneumocystis loads (Babb-Biernacki et al., 2020; Demanche et al., 2019), would further confirm their specific taxonomic status.

This large diversity of Pneumocystis observed in rodents arose from a complex host-parasite evolutionary history, involving a long-lasting process of co-evolution but also several host switches (Latinne et al., 2018; Petružela et al., 2019). Several genera of murid rodents, including the genus Rattus, are known to be infected by several divergent Pneumocystis lineages, sometimes in co-infection (Icenhour et al., 2006; Latinne et al., 2018; Petružela et al., 2019). Our previous study also demonstrated that most Pneumocystis lineages identified in Southeast Asian murid rodents infected several closely related host species, suggesting that the Pneumocystis host specificity in rodents is mostly limited to the generic level (stenoxenism) rather than the species level (monoxenism) (Latinne et al., 2018). These results contradict the apparent strict host specificity observed in other mammals (Akbar et al., 2012; Demanche et al., 2001; Hugot et al., 2003) but some authors recently suggested that the inferred monoxenism of Pneumocystis in some of these studies was due to the inclusion of only distantly related species and the lack of extensive host intrageneric sampling (Babb-Biernacki et al., 2020).

Using Southeast Asian murid and spalacid rodents as models and an extensive dataset, the objectives of this study were to (1) identify the Pneumocystis lineages infecting a large diversity of wild rodents in Southeast Asia and characterize their taxonomic status, host specificity, geographic distribution and genetic structure using both mitochondrial and nuclear markers, (2) identify the rodent species playing a central role in Pneumocystis sharing and transmission in natural rodent communities in Southeast Asia and (3) characterize the environmental conditions and habitat attributes allowing Pneumocystis infection in these rodent populations. In the past decades, Southeast Asia has been exposed to accelerating rates of habitat destruction and modification, such as deforestation, urbanization, and agricultural intensification, with consequent impacts on the structure and richness of host-pathogen communities in the region (Wilcove et al., 2013). Characterized by a high taxonomic diversity of rodents, Southeast Asia is considered to be the center of origin and diversification of Murinae rodents, one of the five subfamilies among murid rodents (Schenk et al., 2013), which display various life histories and collectively inhabit a wide range of ecological niches where they pose significant threats to crop production (Singleton et al., 2010) and for human health as rodents host a high proportion of zoonotic pathogens (Han et al., 2015; Meerburg et al., 2009).

\section{Materials and methods}

\subsection{Study sites and rodent sampling}

Rodent lung samples were collected at multiple sites located in 19 provinces in five countries of Southeast Asia (Thailand, Cambodia, Lao PDR, Philippines and Taiwan) (Fig. 1) as part of the CERoPath and BiodivHealthSEA projects (Herbreteau et al., 2011; Morand et al., 2015). The lung samples were collected immediately after euthanasia and stored in RNAlater (QIAGEN, France) at $-20^{\circ} \mathrm{C}$.

Rodent species were identified in the field using morphological criteria (see www.ceropath.org/references/rodent_field_guide) and molecular barcoding was used to confirm field identification using the protocols described in Latinne et al. (2013) and Pages et al. (2010). The two mitochondrial lineages belonging to Rattus tanezumi were included in this study and referred to as Rattus tanezumi R2 and R3 in accordance with Pages et al. (2010). Rattus tanezumi R2 and R3 correspond to the Rattus rattus Lineage II sensu Aplin et al., 2011 (Aplin et al., 2011) and Rattus rattus Lineage IV sensu Aplin et al., 2011 (Aplin et al., 2011), respectively. Data on the animals used as reference specimens for the barcoding assignment are available on the "Barcoding Tool/RodentSEA" section of the CERoPath project web site (www.ceropath.org).

Rodent species included in this study are neither on the CITES list, nor the IUCN Red List. Animals were treated in accordance with the guidelines of the American Society of Mammalogists, and within the European Union legislation guidelines (Directive 86/609/EEC). Permits from national, regional and local health authorities were obtained before each trapping sessions. Approval notices for rodent trapping and sample collection were provided by the Ministry of Health Council of Medical Sciences, National Ethics Committee for Health Research (NHCHR) in Lao PDR (number 51/NECHR), by the Ethical Committee of Mahidol University, Bangkok, in Thailand (number 0517.1116/661), and by the Animal Care and Use Advisory Committee of National Taiwan Normal University, Taipei, in Taiwan (number 104016). Sample collection from the Philippines was conducted with permission from the Department of Environment and Natural Resources under Wildlife Gratuitous Permit number R4A-WGP-08-2013-LAG-002.

\subsection{Pneumocystis screening}

Samples from 731 rodent individuals belonging to 27 species (Table 1) were screened for the presence of Pneumocystis spp. in their lungs through the amplification of two Pneumocystis mitochondrial genes, the large subunit rRNA (mtLSU rRNA) and the small subunit rRNA (mtSSU rRNA). The 731 samples screened for this study include 445 rodents from a previous study (Latinne et al., 2018) and 286 new individuals, most of them coming from localities and host species not sampled in Latinne et al. (2018). DNA was extracted from lung tissue using the QIAamp DNA mini kit (QIAGEN, France) according to the manufacturer's protocol. The mtLSU rRNA and the mtSSU rRNA 


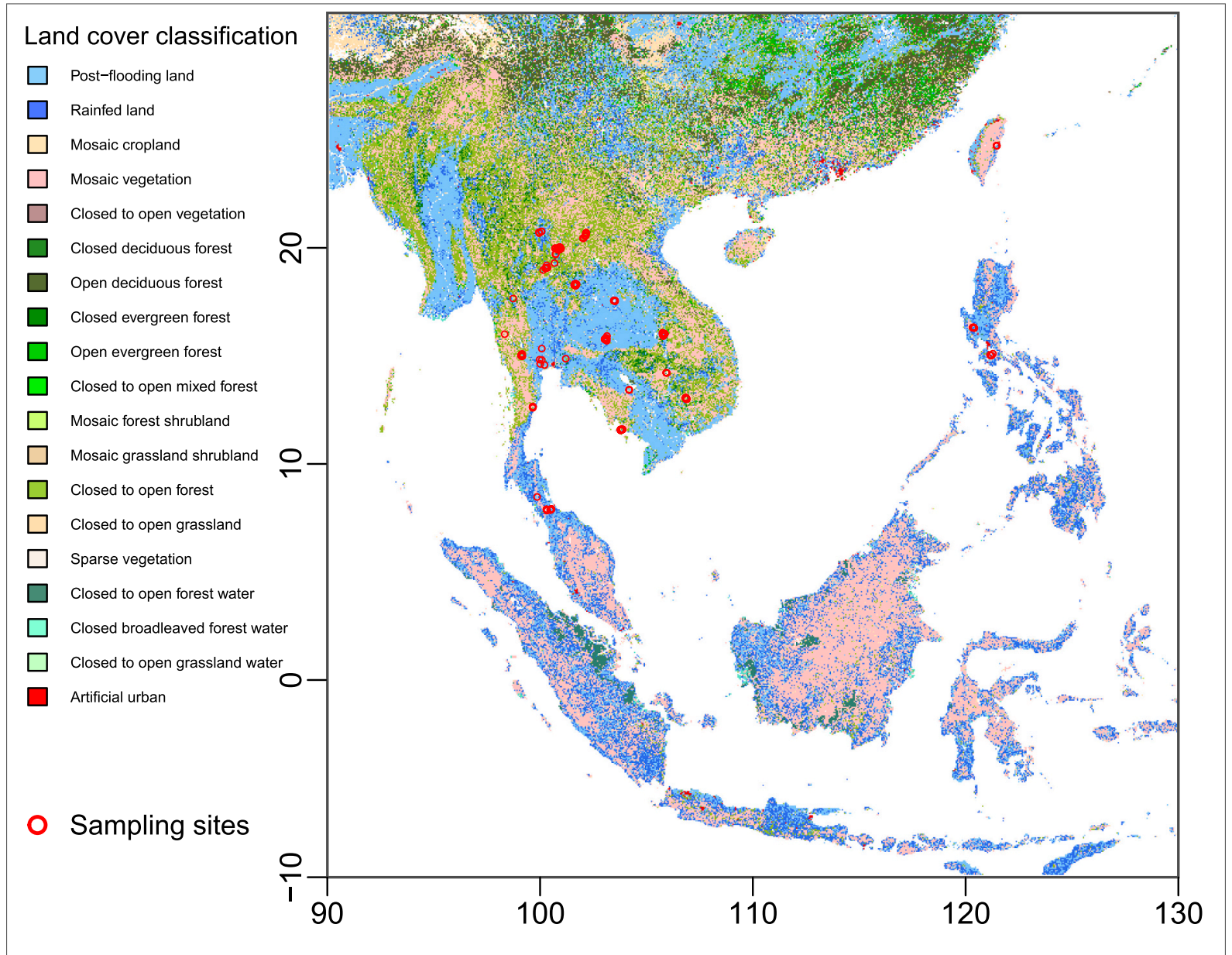

Fig. 1. Southeast Asia land cover map and rodent sampling sites for this study. Land cover map was obtained from the ESA GlobCover 2009 project (http://due.esrin. esa.int/page_globcover.php).

Pneumocystis mitochondrial genes were then amplified by nested PCR using a high-fidelity DNA polymerase (QIAGEN HotStarTaq) and PCR protocols described in Latinne et al. (2018). Samples were considered positive for Pneumocystis infection when a PCR product of the expected size (250-350 bp) was amplified either at mtLSU rRNA or mtSSU rRNA or at both loci. Several negative controls were included in each series of DNA extraction and PCR to detect possible cross-contamination.

A subset of strong positive samples from each host species were selected for PCR amplification of a Pneumocystis nuclear gene, the dihydropteroate synthase (DHPS) gene. A nested PCR using the external primers A HUM $_{\text {( }}$ '-GCG CCT ACA CAT ATT ATG GCC ATT TTA AAT C-3') and B BUM (5'-CAT AAA CAT CAT GAA CCC G-3') (Lane et al., 1997) and internal primers CPRIM (5'-CCC CCA CTT ATA TCA-3') and DPRIM (5'GGG GGT GTT CAT TCA-3') (Demanche et al., 2001) was performed for each sample using a touchdown PCR cycling program as described in Demanche et al. (2001). Sequencing reactions were performed from both ends on an ABI 3730 XL automated DNA sequencer.

\subsection{Phylogenetic analysis}

Sequences were aligned using the ClustalW algorithm and alignments were subsequently refined manually. The mtSSU rRNA hypervariable regions, corresponding to the insertions located at positions 22-354 of the mtSSU rRNA sequence of Pneumocystis carinii (KX257138) and 213-446 of the mtSSU rRNA sequence of Pneumocystis sp. L11 (KX257150) were removed from the alignment as it was not possible to align these regions with other sequences unambiguously, following a strategy similar to the one used in Latinne et al. (2018). Gaps were coded as a fifth state. GenBank Accession numbers of all Pneumocystis sequences generated in this study (MW665237-MW665323, MW694941) and previously published sequences included in our analysis are available in the Supplementary Table S1 Table and Supplementary Table S2.

Phylogenetic trees were obtained using the Maximum Likelihood (ML) inference implemented in PhyML 3.0 (Guindon et al., 2010) and the Bayesian inference implemented in MrBayes 3.2 (Ronquist and Huelsenbeck, 2003) using the mtLSU rRNA (389 bp with indels), mtSSU rRNA (256 bp without hypervariable regions) and DHPS (717 bp) datasets separately and the mtLSU rRNA and mtSSU rRNA concatenated dataset (645 bp with indels). The transition/transversion ratio, the proportion of invariable sites and the gamma distribution parameter of the PhyML analysis were estimated and the substitution model automatically selected (Lefort et al., 2017). The starting tree was determined by BioNJ analysis of the datasets. Robustness of the tree was assessed by 1000 bootstrap replicates. Metropolis-coupled Markov chain Monte Carlo (MCMC) sampling of the Bayesian analysis was performed with 4 chains run for $6.10^{6}$ generations with one tree sampled every 1000 generations, using default parameters as starting values. A $50 \%$ Majority-rule consensus tree was generated after discarding $10 \%$ of trees as burn-in values. All distinct sequence types identified in our dataset using DAMBE (Xia, 2018) were included in these phylogenetic trees. Several Pneumocystis reference sequences were included in the phylogenetic analysis (Supplementary Table S3). Sequences from $P$. jiroveci and Pneumocystis from Macaca sp. were used as outgroups in the DHPS phylogenetic analysis (Supplementary Table S3). We also reconstructed a median-joining network including all Pneumocystis wakefieldiae mtLSU rRNA sequences using Network 10.0 (Bandelt et al., 1999) to assess the 
Table 1

Numbers of tested and Pneumocystis positive individuals for each rodent species.

\begin{tabular}{|c|c|c|}
\hline Family & \multirow{3}{*}{$\begin{array}{l}\text { Number of individuals } \\
\text { tested }\end{array}$} & \multirow{3}{*}{$\begin{array}{l}\text { Number of Pneumocystis positive } \\
\text { individuals }\end{array}$} \\
\hline Tribe & & \\
\hline Species & & \\
\hline \multicolumn{3}{|l|}{ Spalacidae } \\
\hline \multicolumn{3}{|l|}{ Rhizomyni } \\
\hline Cannomys badius & 5 & $5(100 \%)$ \\
\hline Rhizomys & 1 & $1(100 \%)$ \\
\hline \multicolumn{3}{|l|}{ pruinosus* } \\
\hline \multicolumn{3}{|l|}{ Muridae } \\
\hline \multicolumn{3}{|l|}{ Hydromyini } \\
\hline Apomys & 10 & $5(50 \%)$ \\
\hline \multicolumn{3}{|l|}{ banahao* } \\
\hline \multicolumn{3}{|l|}{ Murini } \\
\hline Mus caroli & 12 & $8(66.7 \%)$ \\
\hline Mus pahari* & 5 & $2(40 \%)$ \\
\hline Mus cervicolor & 26 & $10(38.5 \%)$ \\
\hline Mus cookii & 23 & $5(21.7 \%)$ \\
\hline \multicolumn{3}{|l|}{ Rattini } \\
\hline Rattus norvegicus & 58 & $48(82.8 \%)$ \\
\hline Bandicota indica & 27 & $21(77.8 \%)$ \\
\hline Rattus nitidus & 16 & $12(75 \%)$ \\
\hline Sundamys & 9 & $6(66.7 \%)$ \\
\hline \multicolumn{3}{|l|}{ muelleri* } \\
\hline Berylmys bowersi & 8 & $5(62.5 \%)$ \\
\hline Leopoldamys & 5 & $3(60 \%)$ \\
\hline \multicolumn{3}{|l|}{ neilli* } \\
\hline Berylmys berdmorei & 25 & $14(56 \%)$ \\
\hline Niviventer fulvescens & 16 & $8(50 \%)$ \\
\hline Rattus sakeratensis & 25 & $12(48 \%)$ \\
\hline Bandicota savilei & 25 & $11(44 \%)$ \\
\hline Rattus exulans & 138 & $49(35.5 \%)$ \\
\hline Leopoldamys herberti & 12 & $4(33.3 \%)$ \\
\hline Rattus tanezumi R3 & 120 & $39(32.5 \%)$ \\
\hline Rattus tanezumi R2 & 79 & $34(31.6 \%)$ \\
\hline Rattus argentiventer & 17 & $5(29.4 \%)$ \\
\hline Leopoldamys sabanus* & 4 & $1(25 \%)$ \\
\hline Rattus andamanensis & 9 & $2(22.2 \%)$ \\
\hline Rattus tiomanicus* & 19 & $4(21.1 \%)$ \\
\hline Maxomys surifer & 35 & $5(14.3 \%)$ \\
\hline Rattus everetti* & 2 & 0 \\
\hline
\end{tabular}

* Species not included in Latinne et al. (2018).

intraspecific relationships within P. wakefieldiae.

\subsection{Network}

A bipartite network connecting rodent host species $(n=26)$ with all detected Pneumocystis lineages was created using the package tnet (Opsahl, 2007) in R (R Core Team, 2020). Host rodent species and Pneumocystis lineage associations were built on lineage assignments delineated in our phylogenetic trees (see Results). We then transformed this bipartite network into a unipartite network depicting the pattern of shared Pneumocystis parasites by rodent host species using the tnet package in R. The role of each host species within the network was examined using its centrality measurement. A central host node is highly connected to other host nodes and thus is supposed to have a greater transmission potential of Pneumocystis. We calculated the eigenvalue centrality (EC) of each host species using the 'evcent' function from the igraph package (Csardi and Nepusz, 2006) in R.

\subsection{Statistical analysis and environmental data}

Nineteen land use land cover variables (Fig. 1 and Supplementary Table S4) and several environmental and bioclimatic variables (Digital Elevation Model, human population density, normalized difference vegetation index (NDVI), precipitation, and temperature) were extracted from various sources (Supplementary Table S5) for a 500-m buffer around each individual rodent from which samples were collected using the R packages raster (Hijmans et al., 2015), rgdal (Bivand et al., 2019), dismo (Hijmans et al., 2017) and rgeos (Bivand and Rundel, 2017). We then extracted the size of each land use class in each 500-m buffer and computed two additional composite variables, open forest cover (Closed to open forest + Closed to open mixed forest) and mosaic land cover (Mosaic vegetation + Mosaic cropland). We also estimated the fragmentation of the land cover within each buffer by estimating sumFRAC, a metrics calculated using the function PatchStat from the SDMTools package (VanDerWal et al., 2014) in R, which reflects shape complexity across a range of spatial scales and is computed as the logarithm of patch perimeter ( $\mathrm{m}$ ) divided by the logarithm of patch area. All variables were transformed to satisfy normality and homoscedasticity by dividing their size by the total area of the buffer in order to get a percentage of occupation for each variable. The final list of variables observed in the buffer areas around each trapped rodent and used in our models includes: urban cover, forest cover (closed deciduous forest), open forest cover, open vegetation (closed to open vegetation), open grassland (closed to open grassland), mosaic vegetation cover, mosaic land cover, rain fed land cover, waterbodies cover, sumFRAC, temperature, slope, elevation, precipitation, NDVI and human population density.

To assess the links between the probability of Pneumocystis infection in rodents and their environment, we modeled the presence/absence probability of the parasite as a function of these environmental variables using a GLMM with logit function and random effects (for sites) using the package lme4 (Bates et al., 2015) implemented in R. Statistical analyses were performed for all Pneumocystis spp. and then separately for $P$. wakefieldiae in mainland sites (Thailand, Cambodia, Lao PDR) and $P$. wakefieldiae in island sites (Philippines) to assess potential differences in transmission routes of the same Pneumocystis species in different environments. We focused on $P$. wakefieldiae as it was the species for which we had the highest number of positive rodent samples and sampling locations (see Results).

We evaluated support for competing models by investigating the relationship between the occurrence of Pneumocystis parasite and all explanatory variables. We used likelihood-based methods to quantify the alternative models and estimate their parameters. AIC adjusted for sample size (AICc) was used to assess the relative information content of the models. Performance of models was also assessed by estimating the percentage of deviance explained using maximum likelihood R2 and AUC with the pROC (Robin et al., 2011) and MuMIn (Barton and Barton, 2015) packages. Selection of the best competing models was made using package glmulti version 1.0.7 2 (Calcagno and De Mazancourt, 2010) implemented in R, which allows the exploration of all models using automated model selection and model-averaging procedure using a genetic algorithm. Visualization of the predicted response, Pneumocystis infection, was made using the packages sjmisc, sjlabelled and sjPlot (Lüdecke, 2018; Lüdecke and Lüdecke, 2017).

\section{Results}

\subsection{Pneumocystis infection prevalence}

Pneumocystis DNA was detected in 319 samples (43.6\%) belonging to 26 Southeast Asian species; only Rattus everetti yielded no positive sample (Table 1). The six Spalacidae samples belonging to the species Cannomys badius $(5 / 5)$ and Rhizomys pruinosus (1/1) were all positive (100\%) while the prevalence of Pneumocystis infection in Muridae species varied from $14.3 \%(5 / 35)$ in Maxomys surifer to $82.8 \%(48 / 58)$ in Rattus norvegicus (Table 1).

\subsection{Pneumocystis diversity and phylogenetic trees}

We obtained 253 mtLSU rRNA sequences, corresponding to 102 distinct sequence types, and $166 \mathrm{mtSSU}$ rRNA sequences, corresponding to 61 distinct sequence types, after screening 731 rodent samples (Supplementary Table S1). The mitochondrial concatenated dataset (mtLSU rRNA + mtSSU rRNA), excluding some hypervariable and non- 
alignable regions of mtSSU rRNA, included 140 distinct sequence types (Supplementary Table S1). Additionally, we were able to obtain 57 Pneumocystis DHPS sequences, corresponding to 37 distinct sequence types, from 16 rodent host species (Supplementary Table S2).

A total of 16 Pneumocystis phylogenetic lineages, 13 of them infecting Southeast Asian rodents, were identified by taking the topology of the mtLSU rRNA (Supplementary Fig. S1 and S2), mtSSU rRNA (Supplementary Fig. S3 and S4), mitochondrial concatenated (Fig. 2 and Supplementary Fig. S5) and DHPS (Fig. 3 and Supplementary Fig. S6) phylogenetic trees into account. These lineages were named using the same terminology as in Latinne et al. (2018). Co-infection was detected in $15 R$. norvegicus, one $R$. argentiventer, one $N$. fulvescens and one $B$. indica samples whose mtLSU rRNA and mtSSU rRNA sequence types did not belong to the same phylogenetic lineage (Supplementary Table S1). The mitochondrial sequence types from these samples were not concatenated in the combined dataset but were included separately to avoid any phylogenetic misinterpretation in our concatenated trees.

The topologies of the ML (Fig. 2) and Bayesian (Supplementary Fig. S5) mitochondrial concatenated trees were mostly similar. The first lineage to diverge after the outgroup, Lineage 15, included a single highly divergent mtSSU rRNA sequence (HSSU7) isolated in a coinfected $N$. fulvescens sample (CLS14) whose mtLSU rRNA sequence type belonged to Lineage 7 (Pneumocystis from Niviventer sp., Leopoldamys spp. and Maxomys sp.). This highly divergent lineage was also observed in the mtSSU rRNA trees (Supplementary Fig. S3 and S4). A well-supported clade including Lineage 1 (Pneumocystis from wild Danish $R$. norvegicus, referred to as $P c$ f. sp. rattus-tertii in Palmer et al. (2000)), Lineage 2 (Pneumocystis from wild Danish R. norvegicus, referred to as $P c$ f. sp. rattus-quarti in Palmer et al. (2000) and from Southeast Asian Rattus exulans) and Lineage 3 (Pneumocystis from Cannomys sp. and Rhizomys sp.) was next to diverge in the mitochondrial concatenated trees (Fig. 2 and Supplementary Fig. S5). These three lineages were also first to diverge in both mtLSU rRNA phylogenetic trees (Supplementary Fig. S1 and S2) but not in the mtSSU rRNA trees (Supplementary Fig. S3 and S4). Another clade including the remaining 12 lineages among which phylogenetic relationships were poorly resolved was retrieved in the ML and Bayesian mtLSU rRNA and mitochondrial concatenated trees (Fig. 2 and Supplementary Fig. S1, S2 and S5). Lineages 5 and 14 were closely related to a Pneumocystis sequence from cricetid rodent (Microtus) (Lineage 4) in all mitochondrial trees. Lineage 14 included sequences isolated in two $S$. muelleri samples and a co-infected $B$. indica sample whose mtLSU rRNA sequence type belonged to Lineage 11 (Pneumocystis from Bandicota spp.) (Supplementary Table S1). The phylogenetic placement of Lineage 13 (Pneumocystis from Apomys sp.) in the mitochondrial concatenated trees remained unclear as only mtSSU rRNA sequences were available for this lineage. Lineage 13 was nested within Lineage 5 (Pneumocystis from Rattus spp. and Bandicota sp.) in the Bayesian mitochondrial concatenated tree (Supplementary Fig. S5) but corresponded to an independent and divergent lineage in the ML concatenated tree (Fig. 2). The Bayesian and ML mtSSU rRNA trees (Supplementary Fig. S3 and S4) confirmed the phylogenetic independence of Lineage 13 and therefore we considered it as a separate lineage. P. murina (Lineage 8 ) was paraphyletic in the ML mtLSU rRNA tree but was monophyletic in other trees.

Lineage 9 (Pneumocystis carinii), Lineage 10 (Pneumocystis from Berylmys spp.), Lineage 11 (Pneumocystis from Bandicota spp.) and Lineage 12 (Pneumocystis wakefieldiae) were closely related and belonged to a well-supported clade. An additional lineage (Lineage 16) was created to encompass a single mtSSU rRNA sequence (HSSU8) isolated in a coinfected $R$. argentiventer sample (CLS19) whose mtLSU rRNA sequence type belonged to $P$. carinii. This mtSSU rRNA sequence type was related to Lineages 10, 11 and 12 but our mtSSU rRNA phylogenetic trees (Supplementary Fig. S3 and S4) clearly showed that it did not belong to any of these lineages. Co-infections by both $P$. wakefieldiae (Lineage 12) and $P$. carinii (Lineage 9 ) were detected in $15 R$. norvegicus samples characterized by a mtLSU rRNA sequence type closely related to
$P$. carinii while their mtSSU rRNA sequence type was closely related to P. wakefieldiae (Supplementary Table S1).

Most lineages identified in the mitochondrial phylogenetic trees were also retrieved and well-supported in the DHPS tree (Fig. 3). The first Pneumocystis sequences to diverge within the tree ingroup were those from Mus spp. (Pneumocystis murina). However, the DHPS sequence type identified in our Mus pahari sample (HDHPS1) whose mtLSU rRNA and mtSSU rRNA sequences belonged to Lineage 8 (P. murina) did not form a monophyletic clade with the DHPS reference sequence from $P$. murina. DHPS Lineage 7 (including Pneumocystis from Leopoldamys and Niviventer), Lineage 5 (including Pneumocystis from Rattus), Lineage 14 (including Pneumocystis from Sundamys), Lineage 9 (Pneumocystis carinii, including sequences from Rattus) and Lineage 10 (including Pneumocystis from Berylmys) were next to diverge (Fig. 3). The two DHPS sequence types identified in Bandicota indica and corresponding to the mitochondrial Lineage 11 were paraphyletic and closely related to Lineage 12 ( $P$. wakefieldiae) which included DHPS sequences detected in Rattus spp. and Sundamys muelleri. Even after several attempts, we were not able to obtain DHPS sequences from samples belonging to the mitochondrial Lineages 2, 3,13, 15, and 16 .

The only discrepancy between the mitochondrial and nuclear Pneumocystis lineage identification of samples for which both types of markers were obtained was observed for a Rattus norvegicus individual whose both mtLSU rRNA and mtSSU rRNA mitochondrial sequence types clustered within Lineage 9 (P. carinii) while its DHPS sequence type belong to Lineage 12 ( $P$. wakefieldiae). Mitochondrial and nuclear markers yielded similar identification for all other samples. The DHPS sequence type of all $R$. norvegicus samples co-infected by both $P$. wakefieldiae and $P$. carinii according to mitochondrial markers corresponded to $P$. wakefieldiae.

Five of the 13 Pneumocystis mitochondrial linages infecting Southeast Asian rodents are not restricted to a single rodent genus host (Fig. 2). Lineage 3 encompasses Pneumocystis from Cannomys and Rhizomys. Lineage 14 includes Pneumocystis sequences isolated in Sundamys and Bandicota species and is closely related to Lineage 5 which includes Pneumocystis sequences isolated in Rattus and Bandicota species and a sequence identified as $P c$ f. sp. rattus-secundi in wild Rattus norvegicus from Denmark (Palmer et al., 2000). Lineage 7 includes Pneumocystis sequences from Leopoldamys, Maxomys and Niviventer species while sequence types closely related to $P$. wakefieldiae and forming Lineage 12 were identified in several Rattus species but also in Sundamys muelleri. Most of the remaining Pneumocystis lineages identified in several Southeast Asian rodent individuals (Lineages 2, 8, 9, 10 and 11) included sequence types isolated in several congeneric rodent species and were not restricted to a single rodent host species. It is also interesting to note that most Pneumocystis lineages commonly identified in Southeast Asia were distributed in large and mostly overlapping areas and were not restricted to a single region or country (Fig. 4). Lineage 13 (Pneumocystis from Apomys banahao) is restricted to a single location in the Philippines as its host species (Apomys banahao) is endemic to Mt. Banahaw (Heaney et al., 2011) and was not trapped in our other study sites. Lineages 15 and 16 are also restricted to a single location as they were each identified in a single individual.

The median-joining network representing the intraspecific phylogenetic relationships among $P$. wakefieldiae mtLSU rRNA sequence types clearly showed that the most prevalent $P$. wakefieldiae sequence types were shared among several Rattus species and locations (Fig. 5). The mtLSU rRNA sequence types identified in Sundamys muelleri were closely related to $P$. wakefieldiae sequence types identified in Rattus spp. and distinct by only three and seven mutations (Fig. 5A). No clear structure related to host taxonomy was observed, but it is interesting to note that sequence types identified in $R$. exulans were mostly distinct from those identified in other Rattus species. No geographic structure was observed either, with sequences from all locations widespread in the network (Fig. 5B). 


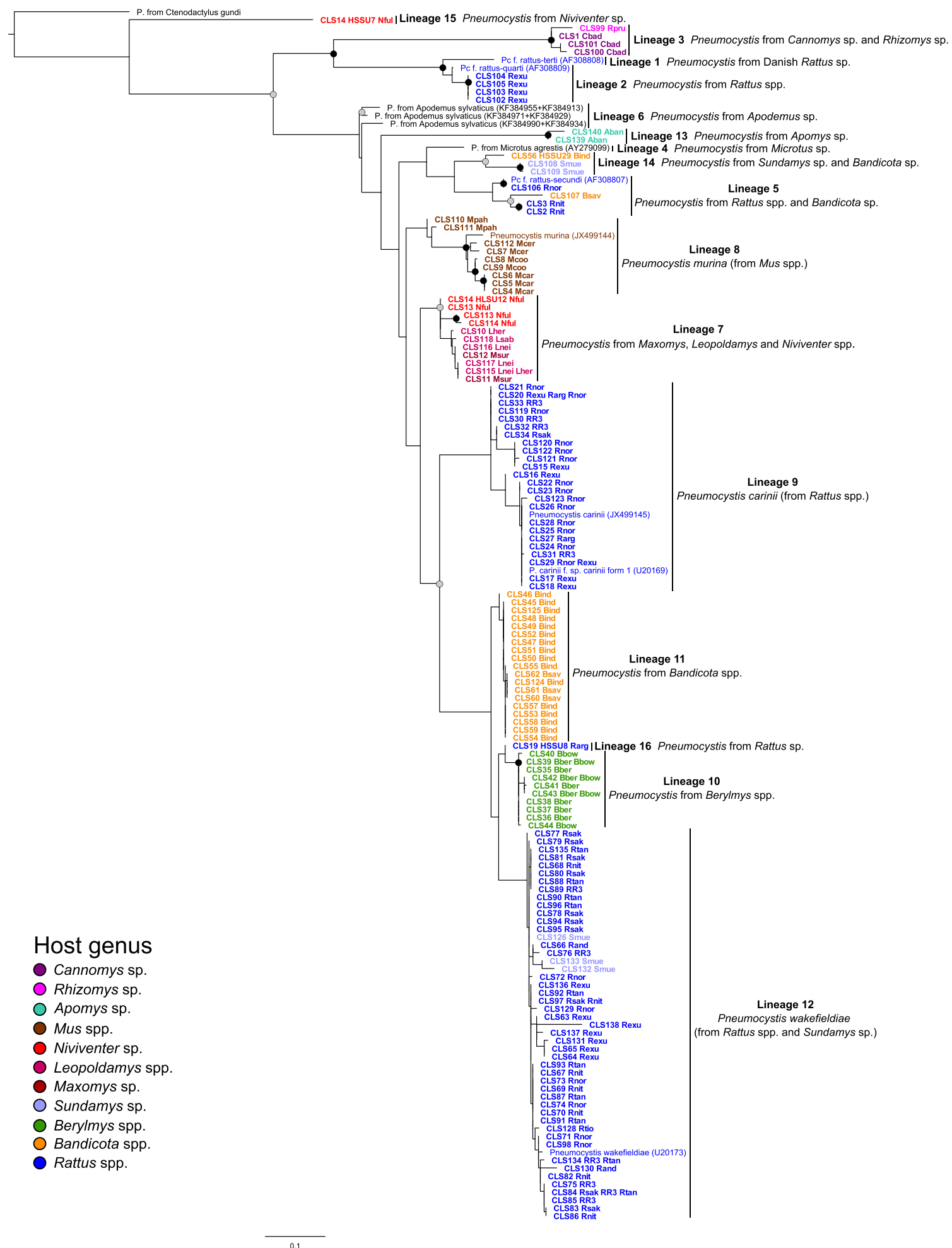

Fig. 2. Maximum Likelihood (ML) tree (GTR + G) depicting phylogenetic relationships among Pneumocystis from Southeast Asian rodents inferred from concatenated mtLSU rRNA and mtSSU rRNA sequences excluding hypervariable regions. Nodes with strong statistical support (bootstrap support $>75 \%$ ) are indicated with a black dot while nodes with moderate statistical support (bootstrap support $>60 \%$ ) are indicated with a grey dot. Node supports from within some lineages were removed for clarity of presentation. Sequences from Southeast Asian rodents are in bold, other reference sequences are from GenBank (accession numbers in brackets). Pneumocystis sequences from rodent genera distributed in Southeast Asia are colored according to their host genus and host species of each sequence type is indicated in the sequence name. Aban: Apomys banahao; Bind: Bandicota indica; Bsav: Bandicota savilei; Bber: Berylmys berdmorei; Bbow: Berylmys bowersi; Cbad: Cannomys badius; Lher: Leopoldamys herberti; Lnei: Leopoldamys neilli; Lsab: Leopoldamys sabanus; Msur: Maxomys surifer; Mcar: Mus caroli; Mcer: Mus cervicolor; Mcoo: Mus cookii; Mpah: Mus pahari; Nful: Niviventer fulvescens; Rarg: Rattus argentiventer; Rand: Rattus andamanensis; Rexu: Rattus exulans; Rnit: Rattus nitidus; Rnor: Rattus norvegicus; Rpru: Rhizomys pruinosus; RR3: Rattus tanezumi R3; Rsak: Rattus sakaretensis; Rtan: Rattus tanezumi R2; Rtio: Rattus tiomanicus; Smue: Sundamys muelleri. 


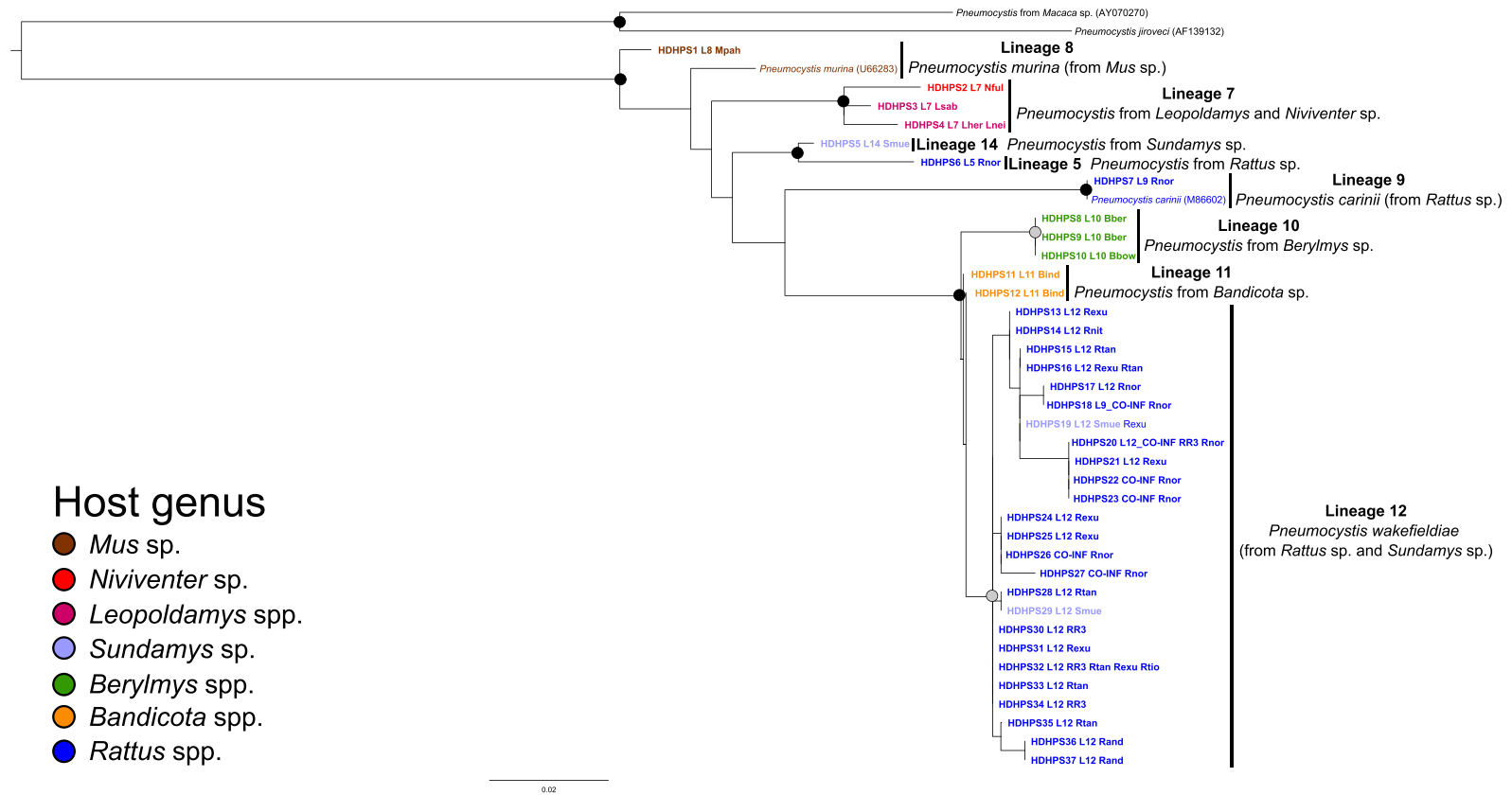

Fig. 3. Maximum Likelihood (ML) tree (HKY85 + G) depicting phylogenetic relationships among Pneumocystis from Southeast Asian rodents inferred from dihydropteroate synthase (DHPS) gene sequences. Nodes with strong statistical support (bootstrap support $>75 \%$ ) are indicated with a black dot while nodes with moderate statistical support (bootstrap support $>60 \%$ ) are indicated with a grey dot. Node supports from within lineages were removed for clarity of presentation. Sequences from Southeast Asian rodents are in bold, other reference sequences are from GenBank (accession numbers in brackets). Pneumocystis lineages from rodent genera distributed in Southeast Asia are colored according to their host genus and host species of each sequence type is indicated in the sequence name. Aban: Apomys banahao; Bind: Bandicota indica; Bsav: Bandicota savilei; Bber: Berylmys berdmorei; Bbow: Berylmys bowersi; Cbad: Cannomys badius; Lher: Leopoldamys herberti; Lnei: Leopoldamys neilli; Lsab: Leopoldamys sabanus; Msur: Maxomys surifer; Mcar: Mus caroli; Mcer: Mus cervicolor; Mcoo: Mus cookii; Mpah: Mus pahari; Nful: Niviventer fulvescens; Rarg: Rattus argentiventer; Rand: Rattus andamanensis; Rexu: Rattus exulans; Rnit: Rattus nitidus; Rnor: Rattus norvegicus; Rpru: Rhizomys pruinosus; RR3: Rattus tanezumi R3; Rsak: Rattus sakaretensis; Rtan: Rattus tanezumi R2; Rtio: Rattus tiomanicus; Smue: Sundamys muelleri.

\subsection{Network and Pneumocystis sharing among rodent species}

The unipartite network describing the interactions among rodent species based on the host species and Pneumocystis lineage associations identified in our phylogenetic trees (Supplementary Table S6) is presented in Fig. 6. Six clusters were clearly identified within the network and only one of them (Cluster 2) included a single rodent host species (Apomys banahao). Two of the remaining clusters (Clusters 1 and 6) included several congeneric species (Berylmys spp.; Mus spp.) while the other three (Clusters 3, 4, and 5) included species from several host genera (Leopoldamys/Niviventer/Maxomys; Rhizomys/Cannomys; Bandicota/Sundamys/Rattus). These clusters were not interacting together, indicating that Pneumocystis transmission was compartmentalized and occurred only between rodent species and genera belonging to the same cluster (Fig. 6).

Most species were characterized by low centrality values $(<0.01)$, except those of Cluster 5 who shared several Pneumocystis lineages (Table 2). Among these species, Rattus norvegicus, Bandicota indica, Bandicota savilei, Rattus nitidus and Sundamys muelleri had the highest centrality values (centrality $>0.7$; Table 2 ) and contributed the most to the sharing of various Pneumocystis lineages among rodent species. Rattus nitidus, Sundamys muelleri and Bandicota savilei were also characterized by a high centrality (Fig. 6 and Table 2).

\subsection{Fine-scale habitat characteristics and Pneumocystis infection}

Of the variables considered in the global GLMM including all Pneumocystis lineages, mosaic land cover and elevation were each important in explaining the Pneumocystis infection probability of rodents according to the best model (Table 3 and Supplementary Fig. S7). A significant positive association $(p=0.037)$ between sites characterized by a patchwork of mosaic of habitats and infection of rodents with any
Pneumocystis lineages was observed (Supplementary Fig. S7). Although the performance of the model is statistically significant with an AUC = 0.64 (0.59-69), the percentage of deviance explained by the best model and estimated by maximum likelihood R2 is low $(\mathrm{R} 2 \mathrm{c}=0.13)$ (Table 3 and Supplementary Fig. S8).

According to the GLMMs including P. wakefieldiae only, sumfrac, a metrics reflecting the fragmentation of the land cover $(p=0.006)$, open forest cover ( $p=0.055)$ and NDVI $(p=0.048)$ were significantly associated to $P$. wakefieldiae infection in mainland sites (Thailand, Cambodia and Lao PDR) while slope was the variable significantly correlated to $P$. wakefieldiae infection in insular sites of the Philippines $(p=0.017)$ (Table 3 and Supplementary Fig. S9). The percentage of deviance explained by these models was estimated by maximum likelihood R2c to 0.38 and 0.35 , respectively, indicating a good prediction of these best models, with good statistical performance given by AUC, respectively $0.80(0.76-0.83)$ and $0.79(0.75-0.83)$ (Table 3 and Supplementary Fig. S8).

\section{Discussion}

\subsection{Pneumocystis diversity and host specificity in murine rodents}

This study further investigates the Pneumocystis evolution, diversity and host specificity in wild rodents from Southeast Asia using both mitochondrial and nuclear markers. Our phylogenies reveal a huge Pneumocystis diversity in murid rodents and do not reflect any global pattern of co-speciation as Pneumocystis lineages detected in spalacid (Lineage 3) and cricetid rodents (Lineage 4) are all closely related to lineages identified in murid rodents (Lineages $1 / 2$ and Lineages 14/5, respectively) while several murid rodent genera (Rattus, Sundamys, Bandicota, and Niviventer) are each infected by two to four highly divergent Pneumocystis lineages characterized by distinct evolutionary 

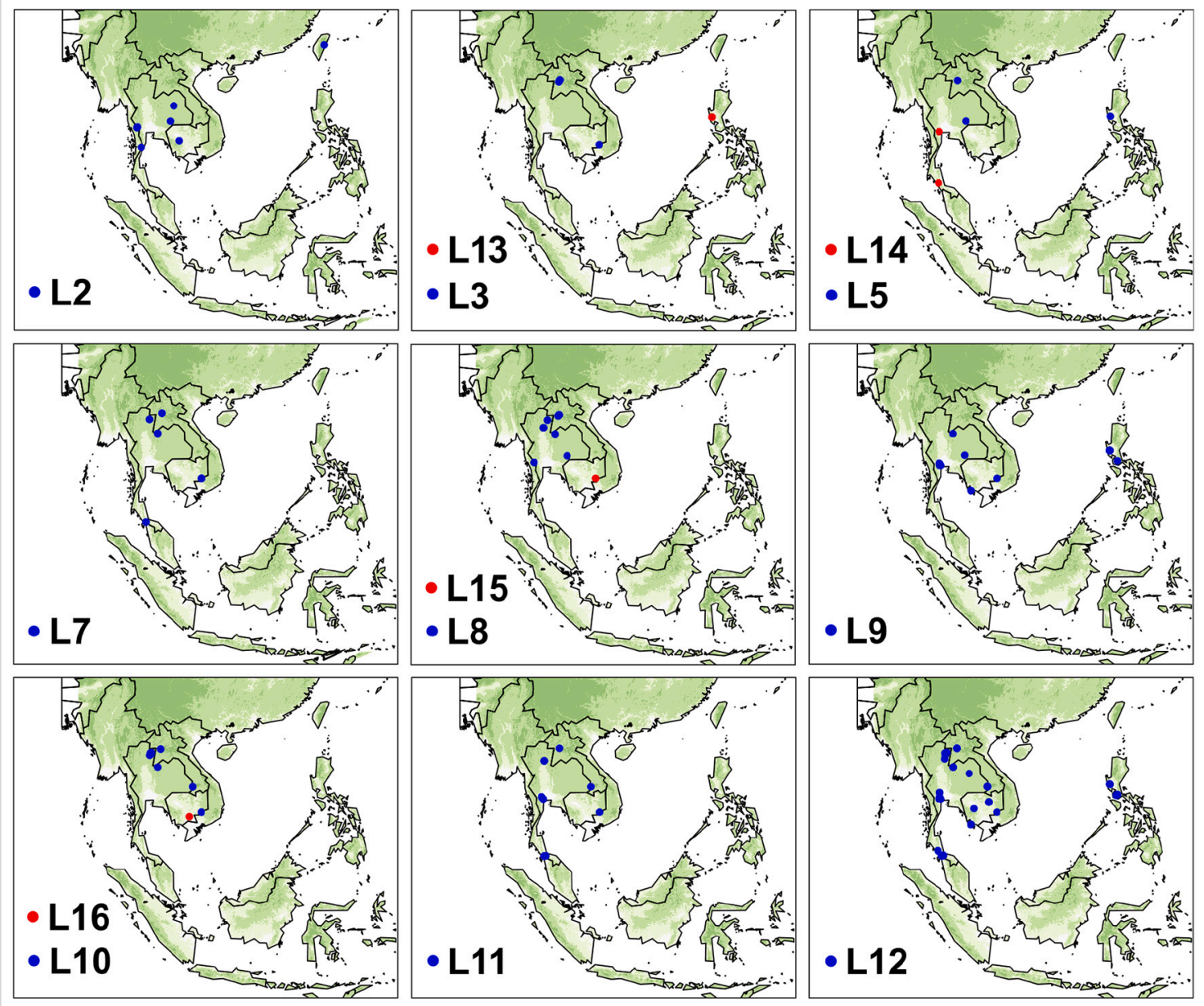

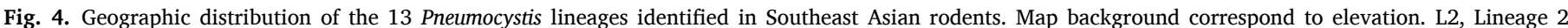

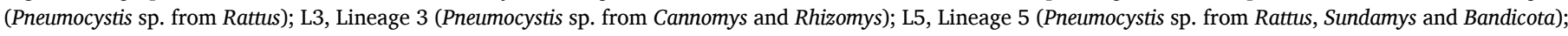

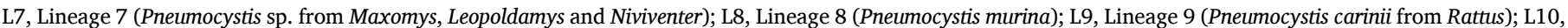

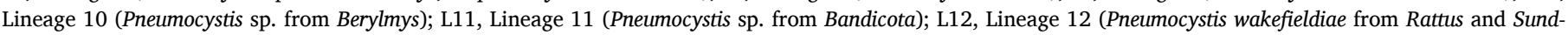

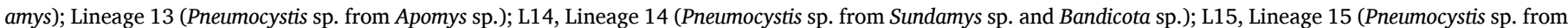
Niviventer sp.); L16, Lineage 16 (Pneumocystis sp. from Rattus sp.)

pathways, revealing a complex evolutionary history of Pneumocystis parasites in rodents.

Our nuclear phylogenetic tree confirmed the phylogenetic and evolutionary independence of most Pneumocystis lineages identified in our mitochondrial phylogenetic trees at both mitochondrial and nuclear markers. The phylogenetic concordance of mitochondrial and nuclear genes confirms the genetic distinctiveness of Lineage 5 (Pneumocystis from Rattus and Bandicota), Lineage 14 (Pneumocystis from Sundamys and Bandicota) and Lineage 7 (Pneumocystis from Maxomys, Leopoldamys and Niviventer) and further suggest their phylogenetic species status according to the Genealogical Concordance Phylogenetic Species Recognition under the Phylogenetic Species Concept (PSC), which uses the phylogenetic concordance of multiple unlinked genes as a criterion to indicate evolutionary independence of lineages and is currently the most widely used species criterion for fungi (Taylor et al., 2000; Giraud et al., 2008). This was also previously indicated by the higher levels of mtDNA genetic distance among these lineages than between other currently recognized Pneumocystis species (Latinne et al., 2018). Lineage 15 is clearly the most divergent of the Pneumocystis lineages identified this study and was detected in a single Niviventer sample, co-infected by Lineage 7. However, this lineage is represented by a single mtSSU rRNA sequence and mtLSU rRNA and DHPS sequences belonging to this lineage are not available. Therefore, additional sequences are needed before we can confirm the specific status of this lineage. Lineage 10 (Pneumocystis from Berylmys) and Lineage 11 (Pneumocystis from Bandicota) are characterized by specific mitochondrial and nuclear sequence types but are less clearly delineated in our nuclear phylogenetic trees where they are closely related to $P$. wakefieldiae (Lineage 12). This pattern could be explained by genetic drift after lineage divergence resulting in these distinctive sequence types but insufficient mutation events to create a pattern of reciprocal monophyly at the slow evolving DHPS gene (Weber et al., 2019). These results do not allow us to confirm the phylogenetic species status of these two lineages. Lineage 16, identified in a $R$. argentiventer sample co-infected by $P$. carinii (Lineage 9), is closely related to these three lineages but its phylogenetic position remains unclear as only one mtSSU rRNA sequence is available. Additional sequences are needed before we can fully characterize this lineage.

A large dataset of sequences from additional nuclear genes would definitely clarify the taxonomic status of each of the lineages identified in this study. However, sequencing of single copy Pneumocystis nuclear genes from wild animals is particularly difficult because of the low fungal load in wild samples and the current lack of reproducible in-vitro culture system of these parasites (Chabé et al., 2010; Cissé and Hauser, 2018; Hauser, 2019). The use of high-throughput sequencing could overcome these difficulties but presents its own technical challenges when applied to Pneumocystis studies (Demanche et al., 2019). High- 

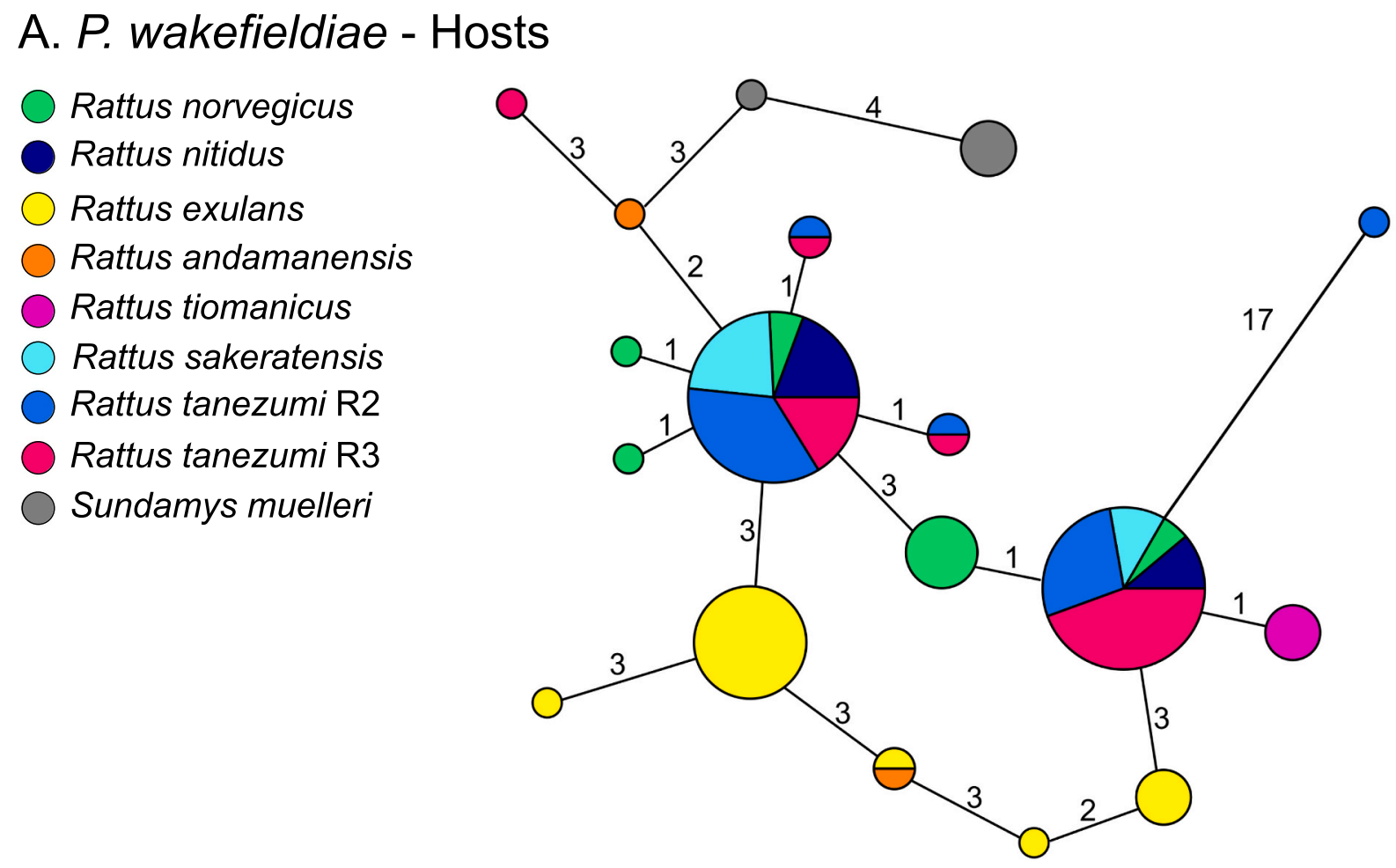

\section{B. P. wakefieldiae - Localities}
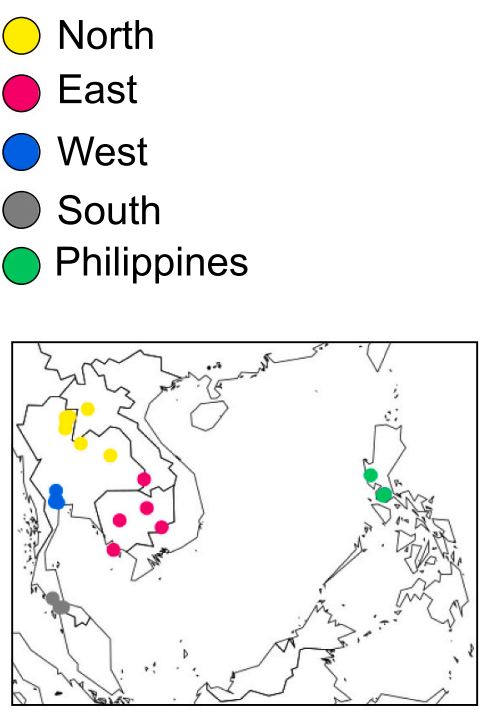

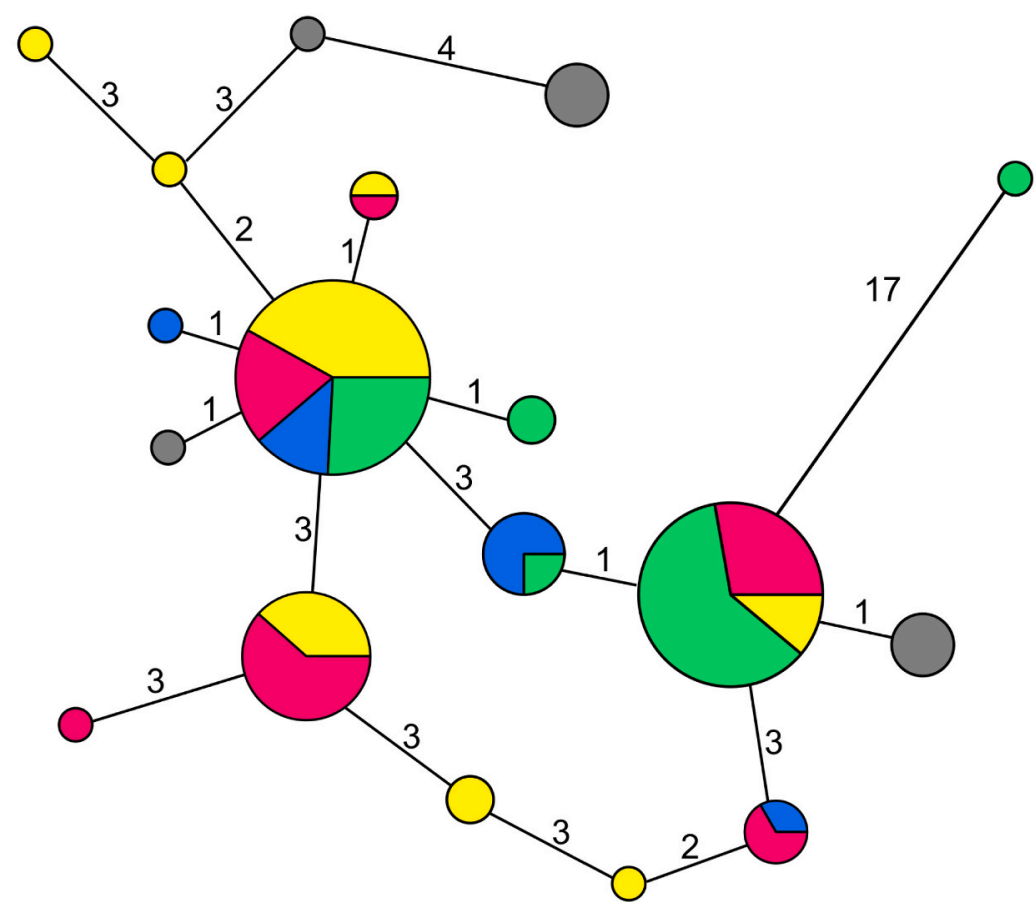

Fig. 5. Median-joining network based on mtLSU rRNA sequences from Pneumocystis wakefieldiae. Circles correspond to distinct sequence types and circle sizes are proportional to the number of animals sharing this haplotype. The number of mutations between sequence types is indicated above branches. Circles are colored according to the rodent host species (A) or sampling localities (B). The insert map shows the rodent sampling localities and their colors correspond to the Philippines and each sampling region within mainland Southeast Asia; North, East, West, South. 


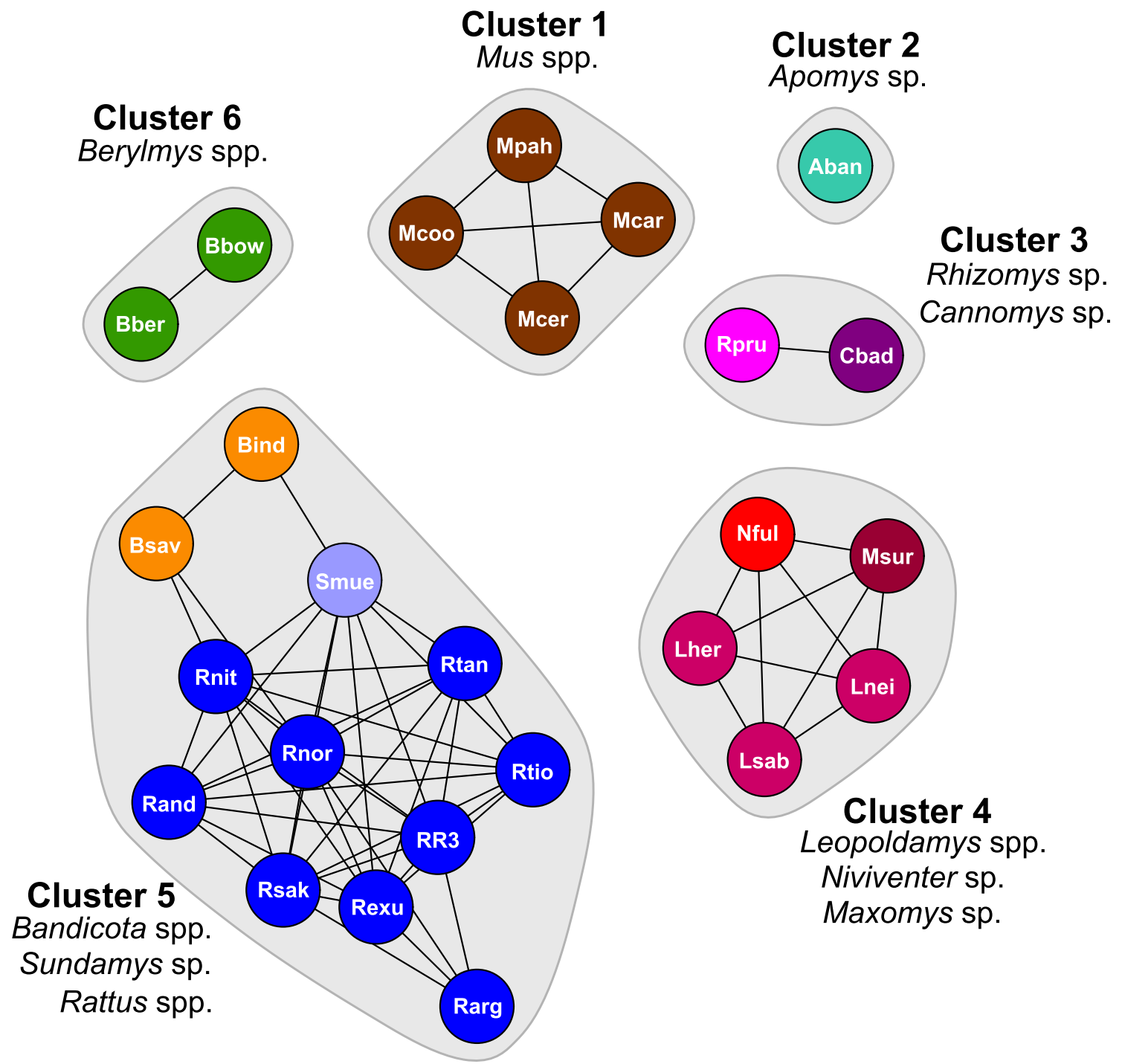

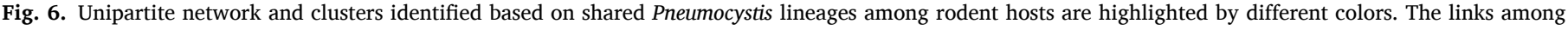

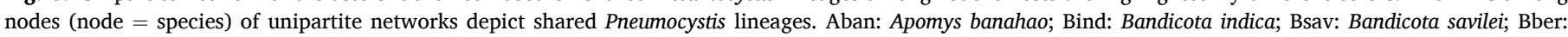

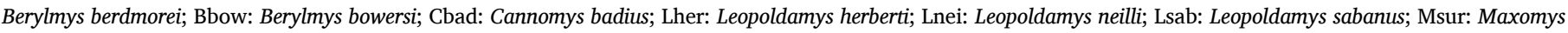

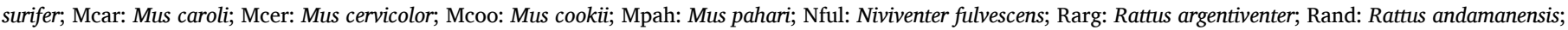

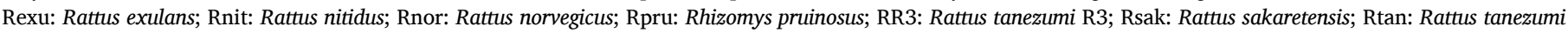
R2; Rtio: Rattus tiomanicus; Smue: Sundamys muelleri.

throughput sequencing would also improve the detection of co-infection of a single host by multiple Pneumocystis lineages as Sanger sequencing may not be able to detect some minority genetic variants. Several instances of co-infections were identified in this study and two of the 13 lineages identified in Southeast Asian rodents (Lineages 15 and 16) were detected only in co-infection with other lineages and seem to be characterized by low prevalence in rodent populations. It is likely that some other rare sequence types were not detected by Sanger sequencing in some samples.

Based on an extended sampling of congeneric rodent host species, including 27 species belonging to 11 genera, this study reveals an even weaker host specificity of Pneumocystis in rodents than suggested by our previous study which included less host species (Latinne et al., 2018). In addition to Lineage 7, which has been detected in three rodent genera, Leopoldamys, Maxomys and Niviventer, by both kinds of genetic markers, we identified four additional Pneumocystis lineages infecting multiple rodent genera. This study provides the first demonstration and compelling evidence of $P$. wakefieldiae (Lineage 12) natural infection in a rodent genus other than Rattus. Indeed, our phylogenetic trees and median-joining network clearly showed that both the mitochondrial and nuclear Pneumocystis sequence types identified in several Sundamys muelleri samples from Thailand are very closely related to $P$. wakefieldiae sequence types identified in multiple Rattus species and belong to the same evolutionary unit. Closely related mitochondrial and nuclear sequence types belonging to Lineages 5 and 14 were also detected in Rattus, Sundamys, and Bandicota. These results are very robust as our mitochondrial findings were confirmed by the nuclear marker used in this study, the DHPS gene. We can exclude the hypothesis that these results could be due to laboratory contaminations as the mtLSU rRNA, mtSSU rRNA and DHPS sequence types isolated in Sundamys and Bandicota and belonging to Lineages 5, 12 and 14 are unique and distinct from those isolated in Rattus and have not been previously isolated in any other samples screened for this study. Finally, the mitochondrial Lineage 3 has also been detected in two spalacid rodent genera, Cannomys and Rhizomys, but these findings have not been confirmed by nuclear genes as we were not able to obtain DHPS sequences from these two genera.

According to the most recent phylogenies of murine rodents where 
Table 2

Network centrality measurement (Eigenvalue centrality values) of each Pneumocystis rodent host species.

\begin{tabular}{ll}
\hline Species & Eigenvalue centrality values \\
\hline Rattus norvegicus & 1 \\
Bandicota savilei & 0.87 \\
Bandicota indica & 0.81 \\
Rattus nitidus & 0.78 \\
Sundamys muelleri & 0.72 \\
Rattus tanezumi R3 & 0.65 \\
Rattus exulans & 0.65 \\
Rattus sakaretensis & 0.65 \\
Rattus argentiventer & 0.38 \\
Rattus tiomanicus & 0.33 \\
Rattus tanezumi R2 & 0.33 \\
Rattus andamanensis & 0.33 \\
Apomys banahao & $<0.001$ \\
Berylmys berdmorei & $<0.001$ \\
Berylmys bowersi & $<0.001$ \\
Cannomys badius & $<0.001$ \\
Leopoldamys herberti & $<0.001$ \\
Leopoldamys neilli Leopoldamys neilli & $<0.001$ \\
Leopoldamys sabanus & $<0.001$ \\
Mus caroli & $<0.001$ \\
Mus cervicolor & $<0.001$ \\
Mus cookii & $<0.001$ \\
Mus pahari & $<0.001$ \\
Maxomys surifer & $<0.001$ \\
Niviventer fulvescens & $<0.001$ \\
Rhizomys pruinosus & $<0.001$ \\
\hline & \\
&
\end{tabular}

the divisions (major evolutionary clades) within each murine tribe were redefined (Rowe et al., 2019), the three genera infected by Lineage 7 belong to two distinct divisions, namely the Maxomys division (including, among others, Maxomys) and the Dacnomys division (including, among others, Leopoldamys and Niviventer). The genera infected by $P$. wakefieldiae (Lineage 12) and Lineages 5 and 14 also belong to distinct divisions, the Bunomys division (including, among others, Sundamys) and the Rattus division (including, among others, Rattus and Bandicota) (Rowe et al., 2019). These findings therefore contradict the strict host specificity paradigm in Pneumocystis often repeated in the literature and demonstrate that the host specificity of Pneumocystis lineages infecting rodents is not restricted to a single host species or even genus but it encompasses much higher taxonomic levels and more distantly related host rodent species than commonly presented in the literature (Demanche et al., 2019; Hauser, 2019).

The divergence between the Dacnomys and Maxomys divisions is dated back to 7.5 Mya, while the Bunomys and Rattus divisions diverged around 3.6 Mya (Rowe et al., 2019), suggesting that the Pneumocystis lineages identified in this study have persisted in these highly distinct rodents without diverging for several million years while maintaining the ability to recognize and evade the immune systems of all these species. Therefore, the Pneumocystis diversity currently observed in murine rodents did not arise from recent co-speciation events along with their hosts. The latest radiations occurring within the murine rodents in Southeast Asia (Rowe et al., 2019) were not followed by Pneumocystis cospeciation and did not lead to the emergence of new Pneumocystis lineages. This was supported by the species divergence time estimates from other studies showing that most Pneumocystis species diverged much before their hosts and did not co-speciate along with them (Ma et al., 2018).

Our findings stress the importance and necessity of using extensive sampling of closely related mammal host species and genera to fully assess the exact host range and host specificity of Pneumocystis species and detect the rarer lineages whose prevalence are lower than the most common lineages. This study also confirms that the proverbial monoxenism and co-evolution of Pneumocystis and their hosts often presented in the literature could be a sampling artifact rather than a biological reality as recently suggested by Babb-Biernacki et al. (2020). Future exhaustive studies of Pneumocystis diversity in taxa other than rodents will help determine whether the relaxed stenoxenism of Pneumocystis in murine rodents observed in this study is an exception or the rule among mammalian hosts.

\subsection{Factors influencing Pneumocystis infection and transmission}

The epidemiology and transmission of Pneumocystis in natural host populations remain poorly understood to date and, to our knowledge, this study is the first to investigate the factors explaining the probability of infection of wild rodents by these airborne-transmitted organisms in their natural habitat. The global Pneumocystis prevalence level observed in this study (43.6\%) is similar to the prevalence found in other wild rodent studies (Danesi et al., 2018; Petružela et al., 2019). Our models suggest that the probability of Pneumocystis infection in rodent hosts is positively correlated to environmental variables reflecting habitat fragmentation and landscape patchiness (i.e. mosaic land, sumfrac and forest open). This correlation is observed both when our models include all Pneumocystis lineages and when only $P$. wakefieldiae on mainland sites is included. These environmental factors may affect Pneumocystis transmission rate and dynamics via impacts on rodent diversity and density. Several studies have shown that the ecological edge effect in fragmented areas and ecosystem boundaries influences both host species richness and population densities (Borremans et al., 2019; Mendoza et al., 2019). As Pneumocystis organisms are directly transmitted via airborne spread of cysts from infected to susceptible hosts without an environmental source (Ma et al., 2018), this increased Pneumocystis prevalence in fragmented habitats may be related to increased contact between rodent individuals in these habitats. A study in bats showed that crowding favored Pneumocystis airborne transmission between bat

Table 3

Results of the best GLMM explaining the occurrence of Pneumocystis in rodents from 19 provinces in Thailand, Lao PDR, Cambodia, Taiwan and the Philippines as a function of surrounding habitat characteristics around individually trapped rodents obtained from land cover layers (see Fig. 1 and Supplementary Table S4).

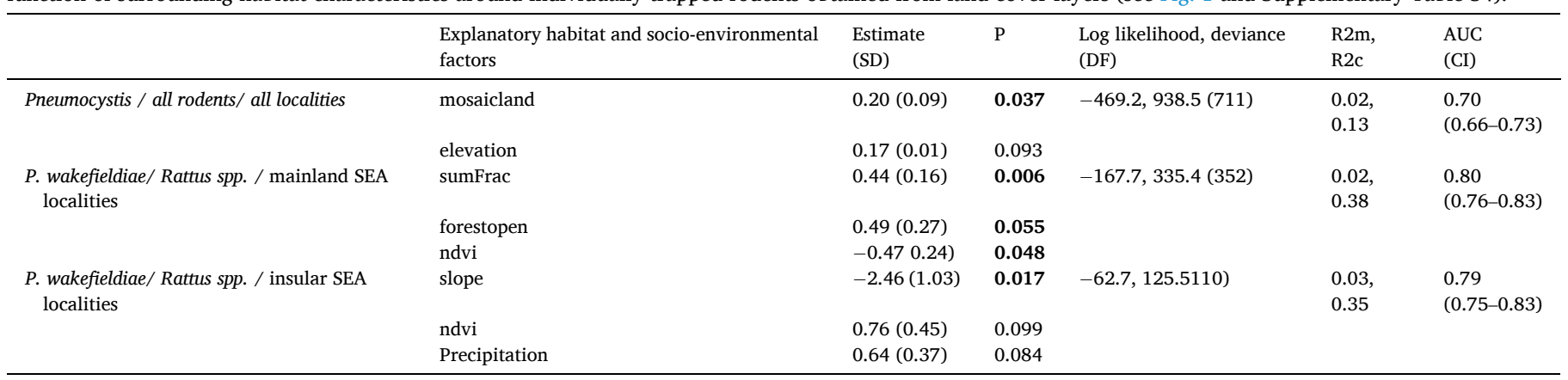

Estimate of the logit function with $\mathrm{SD}=$ standard deviation; residual deviance with $\mathrm{DF}=$ degree of freedom, AUC = accuracy with $95 \%$ confidence interval. $\mathrm{R} 2 \mathrm{~m}$ (marginal) is the variance explained by fixed factors while R2c (conditional) is the variance explained by both fixed and random factors (i.e. the entire model). 
individuals (Akbar et al., 2012). Similar effect of landscape heterogeneity and fragmentation on infection prevalence has been suggested for other parasites and pathogens infecting rodents in various tropical regions of the world (McCauley et al., 2015; Morand et al., 2019; Pumhom et al., 2015; Suzán et al., 2009). Some specific micro-environmental conditions may also favor Pneumocystis infection as we found it is positively correlated to elevation, slope and precipitation levels. An effect of elevation and precipitation on Pneumocystis infection rate was also observed in bats (Akbar et al., 2012) and macaques (Demanche et al., 2003), respectively.

Our network analysis confirmed that the transmission of each Pneumocystis lineage occurred among a small number of rodent species and genera, sometimes phylogenetically distantly related as discussed above, with no interactions among host species belonging to distinct clusters identified in the network. Centrality measurement showed that the rodent species belonging to Cluster 5 and infected by several Pneumocystis lineages, such as several Rattus species but also Sundamys muelleri and Bandicota savilei, contributed the most to the sharing of Pneumocystis lineages among rodent species included in this study. Differences in habitat preference and social structure may explain these findings (Bordes et al., 2007; Bordes et al., 2017). Most of rodent species with high centrality value are synanthropic and habitat-generalist rodents whose abundance increased in fragmented and human-dominated landscapes of mainland Southeast Asia (Morand et al., 2019). Similar to our findings in Pneumocystis, the prevalence of some other pathogens in these species also increased in this type of habitat (Morand et al., 2019). $B$. savilei, B. indica, R. exulans, R. tanezumi, R. sakeratensis, and $R$. norvegicus were already previously identified as bridge host species contributing to most parasite transmission in Southeast Asian ecosystems in a previous study focusing on helminths and several zoonotic pathogens (Bordes et al., 2017). The present study demonstrated that the same synanthropic and generalist rodent species played a similar role of hub in spreading various Pneumocystis lineages in heterogeneous habitats, where they can reach high population densities. Rodent species with a strong preference for forest habitat (Maxomys surifer and Leopoldamys spp.) or dry fields (Berylmys bowersi) (Morand et al., 2019) were infected by a single Pneumocystis lineage and were characterized by very low centrality value in our network and did not significantly contribute to the Pneumocystis cross-species transmission.

Supplementary data to this article can be found online at https://doi. org/10.1016/j.meegid.2021.104978.

\section{Declaration of Competing Interest}

The authors declare that they have no known competing financial interests or personal relationships that could have appeared to influence the work reported in this paper.

\section{Acknowledgements}

We thank the CERoPath team and drivers for their help in the field. Field work was supported by ANR 07 BDIV 012 CERoPath project (Community ecology of rodents and their pathogens in a changing environment in Southeast Asia), ANR 11 CPEL 002 BiodivHealthSEA project (Local impacts and perceptions of global changes: biodiversity, health, and zoonoses in Southeast Asia), ANR-17-CE35-0003-02 FutureHealthSEA (Predictive scenarios of health in Southeast Asia) and CORIGAP (Project 7F-08412.02) funded by the Swiss Agency for Development and Cooperation (SDC). Laboratory work was supported by a Marie Curie COFUND postdoctoral fellowship from the University of Liege to A.L.

\section{References}

Akbar, H., Pinçon, C., Aliouat-Denis, C.-M., Derouiche, S., Taylor, M.-L., Pottier, M., Carreto-Binaghi, L.-H., González-González, A.E., Courpon, A., Barriel, V., Guillot, J.,
Chabé, M., Suarez-Alvarez, R.O., Aliouat, E.M., Dei-Cas, E., Demanche, C., 2012. Characterizing Pneumocystis in the lungs of bats: understanding Pneumocystis evolution and the spread of Pneumocystis organisms in mammal populations. Appl. Environ. Microbiol. 78, 8122-8136.

Aliouat-Denis, C.M., Chabé, M., Demanche, C., Aliouat, E.M., Viscogliosi, E., Guillot, J., Delhaes, L., Dei-Cas, E., 2008. Pneumocystis species, co-evolution and pathogenic power. Infect. Genet. Evol. 8, 708-726.

Aplin, K.P., Suzuki, H., Chinen, A.A., Chesser, R.T., ten Have, J., Donnellan, S.C., Austin, J., Frost, A., Gonzalez, J.P., Herbreteau, V., Catzeflis, F., Soubrier, J., Fang, Y. P., Robins, J., Matisoo-Smith, E., Bastos, A.D.S., Maryanto, I., Sinaga, M.H., Denys, C., Van den Bussche, R.A., Conroy, C., Rowe, K., Cooper, A., 2011. Multiple geographic origins of commensalism and complex dispersal history of black rats. PLoS One 6, e26357.

Babb-Biernacki, S.J., Esselstyn, J.A., Doyle, V.P., 2020. Rethinking host range in Pneumocystis. PLoS Path. 16, e1008824.

Bandelt, H.J., Forster, P., Rohl, A., 1999. Median-joining networks for inferring intraspecific phylogenies. Mol. Biol. Evol. 16, 37-48.

Barton, K., Barton, M.K., 2015. Package 'mumin'. Version 1, p. 18.

Bates, D., Mächler, M., Bolker, B., Walker, S., 2015. Fitting linear mixed-effects models using lme4. J. Stat. Softw. 67, 1-48.

Bivand, R., Rundel, C., 2017. rgeos: interface to geometry engine-open source (GEOS). R package version 0.3-26.

Bivand, R., Keitt, T., Rowlingson, B., Pebesma, E., Sumner, M., Hijmans, R., Rouault, E., Bivand, M.R., 2019. Package 'rgdal'. Bindings for the Geospatial Data Abstraction Library. Available online: https://cran.r-project.org/web/packages/rgdal/index. html. accessed on 15 October 2017.

Bordes, F., Blumstein, D.T., Morand, S., 2007. Rodent sociality and parasite diversity. Biol. Lett. 3, 692-694.

Bordes, F., Caron, A., Blasdell, K., de Garine-Wichatitsky, M., Morand, S., 2017. Forecasting potential emergence of zoonotic diseases in Southeast Asia: network analysis identifies key rodent hosts. J. Appl. Ecol. 54, 691-700.

Borremans, B., Faust, C., Manlove, K.R., Sokolow, S.H., Lloyd-Smith, J.O., 2019. Crossspecies pathogen spillover across ecosystem boundaries: mechanisms and theory. Philos. Trans. R Soc. B 374, 20180344.

Calcagno, V., De Mazancourt, C., 2010. glmulti: an R package for easy automated model selection with (generalized) linear models. J. Stat. Softw. 34, 1-29.

Chabé, M., Dei-Cas, E., Creusy, C., Fleurisse, L., Respaldiza, N., Camus, D., DurandJoly, I., 2004. Immunocompetent hosts as a reservoir of Pneumocystis organisms: histological and RT-PCR data demonstrate active replication. Eur. J. Clin. Microbiol. Infect. Dis. 23, 89-97.

Chabé, M., Herbreteau, V., Hugot, J.P., Bouzard, N., Deruyter, L., Morand, S., Dei-Cas, E., 2010. Pneumocystis carinii and Pneumocystis wakefieldiae in wild Rattus norvegicus trapped in Thailand. J. Eukaryot. Microbiol. 57, 213-217.

Cissé, O.H., Hauser, P.M., 2018. Genomics and evolution of Pneumocystis species. Infect. Genet. Evol. 65, 308-320.

Csardi, G., Nepusz, T., 2006. The igraph software package for complex network research. Int. J. Complex Syst. 1695, 1-9.

Cushion, M.T., Keely, S.P., Stringer, J.R., 2004. Molecular and phenotypic description of Pneumocystis wakefieldiae sp. nov., a new species in rats. Mycologia 96, 429-438.

Danesi, P., da Rold, G., Rizzoli, A., Hauffe, H.C., Marangon, S., Samerpitak, K., Demanche, C., Guillot, J., Capelli, G., de Hoog, S.G., 2016. Barcoding markers for Pneumocystis species in wildlife. Fungal Biol. 120, 191-206.

Danesi, P., Falcaro, C., Ravagnan, S., Da Rold, G., Porcellato, E., Corrò, M., Iatta, R., Cafarchia, C., Frangipane di Regalbono, A., Meyer, W., Capelli, G., 2018. Real-time PCR assay for screening Pneumocystis in free-living wild squirrels and river rats in Italy. J. Vet. Diagn. Investig. 30, 862-867.

Demanche, C., Berthelemy, M., Petit, T., Polack, B., Wakefield, A.E., Dei-Cas, E., Guillot, J., 2001. Phylogeny of Pneumocystis carinii from 18 primate species confirms host specificity and suggests coevolution. J. Clin. Microbiol. 39, 2126-2133.

Demanche, C., Wanert, F., Herrenschmidt, N., Moussu, C., Durand-Joly, I., Dei-Cas, E., Chermette, R., Guillot, J., 2003. Influence of climatic factors on Pneumocystis carriage within a socially organized group of immunocompetent macaques (Macaca fascicularis). J. Eukaryot. Microbiol. 50, 611-613.

Demanche, C., Deville, M., Michaux, J., Barriel, V., Pinçon, C., Aliouat-Denis, C.M., Pottier, M., Noël, C., Viscogliosi, E., Aliouat, E.M., Dei-Cas, E., Morand, S., Guillot, J., 2015. What do Pneumocystis organisms tell us about the phylogeography of their hosts? The case of the Woodmouse Apodemus sylvaticus in Continental Europe and Western Mediterranean Islands. PLoS One 10, e0120839.

Demanche, C., Guillot, J., Chabé, M., 2019. Pneumocystis species co-evolution: state-ofthe-art review. OBM Genet. 3, 1-13.

Frenkel, J.K., 1999. Pneumocystis pneumonia, an immunodeficiency-dependent disease (IDD): a critical historical overview. J. Eukaryot. Microbiol. 46, 89S-92S.

Giraud, T., Refrégier, G., Le Gac, M., de Vienne, D.M., Hood, M.E., 2008. Speciation in fungi. Fungal Genet. Biol. 45, 791-802.

Guindon, S., Dufayard, J.F., Lefort, V., Anisimova, M., Hordijk, W., Gascuel, O., 2010. New algorithms and methods to estimate maximum-likelihood phylogenies: assessing the performance of PhyML 3.0. Syst. Biol. 59, 307-321.

Han, B.A., Schmidt, J.P., Bowden, S.E., Drake, J.M., 2015. Rodent reservoirs of future zoonotic diseases. Proc. Natl. Acad. Sci. 112, 7039-7044.

Hauser, P.M., 2019. Is the unique camouflage strategy of Pneumocystis associated with its particular niche within host lungs? PLoS Path. 15, e1007480.

Heaney, L.R., Balete, D.S., Rickart, E.A., Alviola, P.A., Duya, M.R.M., Duya, M.V., Veluz, M.J., VandeVrede, L., Steppan, S.J., 2011. Chapter 1: seven new species and a new subgenus of forest mice (Rodentia: Muridae: Apomys) from Luzon Island. Fieldiana Life Earth Sci. 1-60. 
Herbreteau, V., Jittapalapong, S., Rerkamnuaychoke, W., Chaval, Y., Cosson, J.-F., Morand, S., 2011. Protocols for field and laboratory rodent studies. Retrieved from. https://hal.ird.fr/ird-00714514.

Hijmans, R.J., Van Etten, J., Cheng, J., Mattiuzzi, M., Sumner, M., Greenberg, J.A., Lamigueiro, O.P., Bevan, A., Racine, E.B., Shortridge, A., 2015. Package 'raster'. R package 734 .

Hijmans, R.J., Phillips, S., Leathwick, J., Elith, J., Hijmans, M.R.J., 2017. Package 'dismo'. Circles 9, 1-68.

Hugot, J.P., Demanche, C., Barriel, V., Dei-Cas, E., Guillot, J., 2003. Phylogenetic systematics and evolution of primate-derived Pneumocystis based on mitochondrial or nuclear DNA sequence comparison. Syst. Biol. 52, 735-744.

Icenhour, C.R., Arnold, J., Medvedovic, M., Cushion, M.T., 2006. Competitive coexistence of two Pneumocystis species. Infect. Genet. Evol. 6, 177-186.

Keely, S.P., Fischer, J.M., Cushion, M.T., Stringer, J.R., 2004. Phylogenetic identification of Pneumocystis murina sp. nov., a new species in laboratory mice. Microbiology 150 1153-1165.

Lane, B.R., Ast, J.C., Hossler, P.A., Mindell, D.P., Bartlett, M.S., Smith, J.W., Meshnick, S. R., 1997. Dihydropteroate synthase polymorphisms in Pneumocystis carinii. J. Infect. Dis. $175,482-485$.

Latinne, A., Waengsothorn, S., Rojanadilok, P., Eiamampai, K., Sribuarod, K., Michaux, J. R., 2013. Diversity and endemism of Murinae rodents in Thai limestone karsts. Syst. Biodivers. 11, 323-344.

Latinne, A., Bezé, F., Delhaes, L., Pottier, M., Gantois, N., Nguyen, J., Blasdell, K., DeiCas, E., Morand, S., Chabé, M., 2018. Genetic diversity and evolution of Pneumocystis fungi infecting wild Southeast Asian murid rodents. Parasitology 145, 885-900.

Lefort, V., Longueville, J.-E., Gascuel, O., 2017. SMS: smart model selection in PhyML. Mol. Biol. Evol. 34, 2422-2424.

Lüdecke, D., 2018. sjmisc: data and variable transformation functions. J. Open Source Softw. 3, 754.

Lüdecke, D., Lüdecke, M.D., 2017. Package 'sjPlot'.

Ma, L., Cissé, O.H., Kovacs, J.A., 2018. A molecular window into the biology and epidemiology of Pneumocystis spp. Clin. Microbiol. Rev. 31 e00009-00018.

McCauley, D.J., Salkeld, D.J., Young, H.S., Makundi, R., Dirzo, R., Eckerlin, R.P., Lambin, E.F., Gaffikin, L., Barry, M., Helgen, K.M., 2015. Effects of land use on plague (Yersinia pestis) activity in rodents in Tanzania. Am. J. Trop. Med. Hygiene 92, 776-783.

Meerburg, B.G., Singleton, G.R., Kijlstra, A., 2009. Rodent-borne diseases and their risks for public health. Crit. Rev. Microbiol. 35, 221-270.

Mendoza, H., Rubio, A.V., García-Peña, G.E., Suzán, G., Simonetti, J.A., 2019. Does landuse change increase the abundance of zoonotic reservoirs? Rodents say yes. Eur. J. Wildl. Res. 66, 6.

Morand, S., Bordes, F., Blasdell, K., Pilosof, S., Cornu, J.-F., Chaisiri, K., Chaval, Y., Cosson, J.F., Claude, J., Feyfant, T., Herbreteau, V., Dupuy, S., Tran, A., 2015. Assessing the distribution of disease-bearing rodents in human-modified tropical landscapes. J. Appl. Ecol. 52, 784-794.

Morand, S., Blasdell, K., Bordes, F., Buchy, P., Carcy, B., Chaisiri, K., Chaval, Y., Claude, J., Cosson, J.-F., Desquesnes, M., Jittapalapong, S., Jiyipong, T., Karnchanabanthoen, A., Pornpan, P., Rolain, J.-M., Tran, A., 2019. Changing landscapes of Southeast Asia and rodent-borne diseases: decreased diversity but increased transmission risks. Ecol. Appl. 29, e01886.

Opsahl, T., 2007. thet: Software for Analysis of Weighted, Two-Mode, and Longitudinal Networks. R package.

Pages, M., Chaval, Y., Herbreteau, V., Waengsothorn, S., Cosson, J.F., Hugot, J.P. Morand, S., Michaux, J., 2010. Revisiting the taxonomy of the Rattini tribe: a phylogeny-based delimitation of species boundaries. BMC Evol. Biol. 10, 184.

Palmer, R.J., Settnes, O.P., Lodal, J., Wakefield, A.E., 2000. Population structure of ratderived Pneumocystis carinii in Danish wild rats. Appl. Environ. Microbiol. 66, 4954-4961.

Petružela, J., Bryja, J., Bryjová, A., Katakweba, A., Sabuni, C., Baird, S.J.E., de Bellocq, J. G., 2019. Evolutionary history of Pneumocystis fungi in their African rodent hosts. Infect. Genet. Evol. 75, 103934.

Pumhom, P., Morand, S., Tran, A., Jittapalapong, S., Desquesnes, M., 2015. Trypanosoma from rodents as potential source of infection in human-shaped landscapes of SouthEast Asia. Vet. Parasitol. 208, 174-180.

R Core Team, 2020. R: A Language and Environment for Statistical Computing. R Foundation for Statistical Computing, Vienna, Austria.

Robin, X., Turck, N., Hainard, A., Tiberti, N., Lisacek, F., Sanchez, J.-C., Müller, M., 2011. pROC: an open-source package for $\mathrm{R}$ and $\mathrm{S}+$ to analyze and compare ROC curves. BMC Bioinformat. 12, 1-8.

Ronquist, F., Huelsenbeck, J.P., 2003. MrBayes 3: Bayesian phylogenetic inference under mixed models. Bioinformatics 19, 1572-1574.

Rowe, K.C., Achmadi, A.S., Fabre, P.-H., Schenk, J.J., Steppan, S.J., Esselstyn, J.A., 2019. Oceanic islands of Wallacea as a source for dispersal and diversification of murine rodents. J. Biogeogr. 46, 2752-2768.

Schenk, J.J., Rowe, K.C., Steppan, S.J., 2013. Ecological opportunity and incumbency in the diversification of repeated continental colonizations by muroid rodents. Syst. Biol. 62, 837-864.

Singleton, G.R., Belmain, S., Brown, P.R., Aplin, K., Htwe, N.M., 2010. Impacts of rodent outbreaks on food security in Asia. Wildl. Res. 37, 355-359.

Suzán, G., Marcé, E., Giermakowski, J.T., Mills, J.N., Ceballos, G., Ostfeld, R.S., Armién, B., Pascale, J.M., Yates, T.L., 2009. Experimental evidence for reduced rodent diversity causing increased hantavirus prevalence. PLoS One 4, e5461.

Taylor, J.W., Jacobson, D.J., Kroken, S., Kasuga, T., Geiser, D.M., Hibbett, D.S., Fisher, M.C., 2000. Phylogenetic species recognition and species concepts in fungi. Fungal Genet. Biol. 31, 21-32.

VanDerWal, J., Falconi, L., Januchowski, S., Shoo, L., Storlie, C., 2014. SDMTools: species distribution modelling tools: tools for processing data associated with species distribution modelling exercises. $\mathrm{R}$ package version 1, pp. 1-221.

Weber, A.A.-T., Stöhr, S., Chenuil, A., 2019. Species delimitation in the presence of strong incomplete lineage sorting and hybridization: lessons from Ophioderma (Ophiuroidea: Echinodermata). Mol. Phylogenet. Evol. 131, 138-148.

Wilcove, D.S., Giam, X., Edwards, D.P., Fisher, B., Koh, L.P., 2013. Navjot's nightmare revisited: logging, agriculture, and biodiversity in Southeast Asia. Trends Ecol. Evol. $28,531-540$.

Xia, X., 2018. DAMBE7: new and improved tools for data analysis in molecular biology and evolution. Mol. Biol. Evol. 35, 1550-1552. 